and much to my satisfaction, the patient grew steadily better. It was my privilege to see her one week ago, and she was in very good condition. I should like to ask Dr. Tuttle if he has observed contractures or strictures after the water treatment. I do not know whether the stricture in my case is due to the disease or to the topical applications made by me prior to the time of beginning the ice water treatment. The patient I refer to is now perfectly well except for a contracture in the rectum. Previously, Tuttle's proctoscope could be used, i. e., passed without pain, but now this is impossible; the stricture will only admit a medium large size old style Kelly's cystoscope.

Dr. Samuel S. Gant, New York-Was not Dr. Weir the first to suggest this opening of the appendix and using it for irrigating the colon?

Dr. William Krauss, Memphis, Tenn.-In reference to the diagnosis of amebic dysentery, $I$ think $I$ am right in stating that $I$ never made a diagnosis unless my microscope and the patient were in the room together. I have never been able to revive a motionless ameba. The mucus from the discharge or swabbing should be transferred quickly to a warm slide. I have tried Dr. Tuttle's suggestion of cold water and with suecess. Water ean not be used hot enough to injure the parasite.

DR. TutTLE-As stated in the paper it was Dr. Weir who first suggested making use of the appendix for this purpose. I modified Dr. Weir's operation by drawing out the appendix and absolutely getting rid of it, at the same time obtaining access to the seat of the amebic infection. I omitted to speak of the mixed infections associated with dysentery of this type. I also wished to speak of the importance of the local treatment of ulcerations down in the rectum and in the sigmoid, when using cold water irrigations; and of hot water for improving the circulation. There are certain cases in which the ulcers become infected with a secondary infection, and they must be treated individually by local applications of tincture of iodin, etc. With regard to ipecac and quinin, how can they affect the buried amebæ? The amebæ are not in the blood, but in the submucosa. The only way to reach them in this position is by the use of low temperatures. Dr. Morton of New York has recently reported a case in which the diagnosis of amebic dysentery was made and the case was treated by him by radiized quinin, and he claims the patient is well. Operation is to be resorted to only when all other methods of cure have failed. I do not believe it is a dangerous operation; it is certainly not more dangerous than leaving the amebre to progress to a chronic state, with its dangerous complications. There is little danger in pulling up the appendix and holding it there until adhesions form and shut off the peritoneal cavity, and then amputating it.

\section{THE PATHOLOGY AND ETIOLOGY OF ARTHRITIS DEFORMANS.*}

\section{THOMAS MCCRAE, M.D. BALTIMORE.}

In discussing this subject there are two courses open; one is to go over the various theories and ideas that have been put forth, the other is to dispense with tradition so far as we can and state as closely as possible the condition of our knowledge in regard to this subject to-day. It is perhaps well to state frankly that we are more likely to outline our ignorance than to state our knowledge, but we can at any rate determine the lines in which investigation is most likely to be profitable, because I take it that no one would be likely to say to-day that we had

*Read at the Fifty-fifth Annual Session of the American MedIral Association, in the Section on Practice of Medicin... and ap proved for publication by the Executive Committee: Drs. J. $M$ Anders, Frank Jones and W. S. Thayer. anything very firmly established in regard to the subject of this paper.

'Through bacteriologic work we have been able to limit certain forms of arthritis definitely. It would seem best to restrict the term "infective arthritis" to those forms in which we have a distinct causal agent proved. The forms associated with the tubercle bacillus, gonococcus, pneu. mococcus, etc., belong to this group. Recent work sug. gests that acute articular rheumatism will before long bo added to these. In regard to arthritis deformans, the evidence is as yet not positive, but very suggestive.

\section{GENERAI CONSTDERATIONS.}

Almost every point that one can raise in regard to this disease is a disputed one. The more important ones seem to me as follows:

1. Have we under this term one disease with many different manifestations, or have we included two, perhaps more, totally distinct diseases?

The decision in regard to this point is, of course, essential before onc can get to any satisfactory conclusion as to the etiology or pathology, and I trust the discus. sion may clear up some of the points in regard to it. As has been frcquently pointed out, there are certainly varying types of the disease. Some observers have distinguished these as the rheumatoid or atrophic type, and the osteoarthritic or hypertrophic type. In the former the disease may occur at any age from 2 to 70 years; the arthritis is usually polyarticular, may come on with great suddenness and recur perhaps at long intervals, or it may be a slow, progressive process. In this type the changes are more especially atrophic and often especially periarticular. As has been shown by Goldthwait, there is much increased excretion of calcium salts. In the seconi or osteoarthritic group there is in some ways a rather different picture; the classical description of the older writers, which laid special stress on the production of osteophytic growth, applies especially to these. These groups will be considered more in detail later.

2. The question of relationship of arthritis deforman: to acute rheumatism. Of course, it is quite possible that a patient may have acute articular rheumatism and years afterward arthritis deformans, but I believe this is the only association between the two diseases, and that arthritis deformans has nothing whatever to do with acute articular rheumatism. Much of the confusion has been due to the mistake of considering attacks of arthritis deformans as acute articular rheumatism. Of course, this opinion is contrary to that of many of the authorities on the disease.

3. The question as to the essential etiologic factor. which we have to discuss under three heads: (a) A neural cause; (b) infection, with a definite specific organism; (c) infection, with various organisms.

One point has to be strongly kept in mind, that although the early and late conditions are quite different both clinically and pathologically, nearly all the older descriptions of the disease are based on the late changes, and no account is taken of the early ones. This has resulted in the process being commonly regarded as a degenerative one only. It would be as reasonable to base all our views as to the pathology of chronic nephritis on the conditions found in a much contracted kidney, ignoring the early stages.

Arthritis deformans appears under very different conditions and in varying forms; coming on in the first decade of life, it differs very much from the form seen in adult life. In one it may be very acute, advancing rapidly to extreme deformity and destruction of the joints. 
or it may be exceedingly chronic and remain quiescent for a long periud of time. Does this necessarily imply the existence of distinct diseases? A comparison with tuberculosis should suggest caution in passing any such judgment. Consider the varying manilestations of tuberculosis at different ages. Our knowledge of tuberculosis not so long ago was about as chaotic as of arthritis deformans to-day, and before the discovery of the tubercle bacillus it probably seemed about as hopeless to satisfactorily explain all the conditions as it is to-day in regard to arthritis deformans.

There are some points of the clinical aspect of the disease that may be noted here. One is that the more carefully the histories of the cases are studied the stronger is the impression that in the great majority there have been acute features at some time or other. It may have been many years before. This is true of both types of the disease. The man with bony deposits in the spine may hare acute recurring attacks exactly as in the polyarticular form, which may begin with symptoms suggesting acute articular rheumatism. Changes in the early stages we have had comparatively little chance to study pathologically. It is to be hoped that no opportunities for autopsies on early cases will be lost. These are most likely to occur in persons who have died of accident or of some other disease.

There is one point to which I should like to draw special attention, namely, that the changes in arthritis deformans are not necessarily progressive. We have been too apt to regard the condition as going on to marked degeneration and destruction, but I believe the more carefully the disease is studied the more instances we will find of patients in whom it is to all intents and purposes cured. There is a certain amount of damage left in the joints. but this is comparatively slight and gives no great disability. This, of course, corresponds in general to our ideas of infectious disease. Wre have types from the very mild to the very severe. Take, for example, the varying types of virulence of gonorrheal arthritis-in one case slight and of no very great severity, and another going on to almost complete destruction of the joint. but the average case with a tendency to chronicity and the establishment of more or less change. Have we not practically a parallel condition in arthritis deformans?

\section{PATHOLOGIC ANATOMY}

It is convenient in some ways in discussing this to take up the features of the two main types of the disease separately. An important matter to settle is the association of these two conditions. That they occur together in the same patient $I$ think there can be no doubt. and this is evident both from clinical evidence and from the radingraphs. It is, however, more convenient to consider them separately. and the terms atrophic. and hypertrophic are convenient to use. This is convenient for description. whether they be considered as varying types or distinct diseases.

The Atronhic Type.-This, which is often termed "rheumatoid," corresponds in the majority of cases to the polyarticular type. The patholngic changes seem to follow a fairly definite course. The first structure to show a change is apparently the svnovial membrane. If the joint be opened in an early case the capsule is usually found to be redder than normal; the surface may have a velvet appearance, or perhaps be covered by dark red material, much like granulation tissue. This granalation-like material in certain situations apparently replace the synovial membrane and creeps over the sur- face of the cartilage; in the carpus this process may advance to great destruction of interosseous structures. As the process continues the synovial membrane usually becomes thickened; various changes may occur, especially in the line of proliferation, the fringes becoming larger and at times showing the so-called vilious arthritis. These fringes may become detached and form foreign bodies in the joint. These in some instances may even become calcified.

That there is any definite histologic picture which is characteristic of the changes in the synovial membrane in this disease does not seem likely. The condition seems to be much alike in the various more or less chronic inflammations of the joints. In the earlier acute stages the membrane shows infiltration of a very large number of mononuclear cells, often collected irregularly. and these sometimes, when on the surface of the membrane, may lead to the naked-eye diagnosis of tubercle In the later more chronic stages these colls become less numerous, there is considerably more fibrous tissue, and the small arteries show obliterating endarteritis. The same condition can, however, be seen in other joint inflammations and is not peculiar to arthritis deformans. It is quite probable that in certain cases there may be no further change than that mentioned in the capsule and a fair degree of recovery take place.

In many instances, however, after the changes in the synovial membrane, the cartilage is attacked. The first change in the cartilage is usually erosion, and this, as in the case reported by Dr. Hale White, ${ }^{1}$ seemed to be determined by the condition of the synovial membrane near it. Pressure of the synovial fringes seems to determine the perforation of the cartilage. The amount of destruction of the cartilage varies greatly. It may be comparatively slight, and is often very irregular in extent. With this there may be very little damage to the bone, although the skiagraphs in some cases suggest that there is very frequently atrophy of the bone in the neighborhood of the joint. The bone may, however. show foci of inflammation, and in these areas there is comparative absence of fat cells.

The fluid found in the joints is usually somewhat turbid; the cells found do not seem to hare any special significance. With these changes in the joint itself the structures about it are also involved. There is inflammation and infiltration of the periarticular structures. These become adherent to the symovial membrane, and there may be considerable swelling. Ankylosis proper of the joint rarely results The subsequent course varies very greatly. The process may cease to advance, ab. sorption gradually occur, and the joint left little damaged, but more susceptible to injury on subsequent attacks. Or, on the contrary, it may go on to almost complete destruction and be useless for further function. With this, of course, other conditions are associatedmuscular atropliy, contractures and subluxation of the joint. In some instances the joint cavity may become practically obliterated.

What explanation can be given for such conditions? When we consider the many changes found in infective joint disease, such varying results seem perfectly possible as the effect of a specific bacterial infection. The action of toxins may be suggested, but as to this we know little. That they may influence the production of an arthritis is suggested by the cases following the injection of diphtheria antitoxin.

It is possible that various septic conditions may be

\footnotetext{
1. Guv's Hospital Reports, vol. Ivil.
} 
causal; at any rate, the influence of intercurrent septic infections in this disease is well known. Thus in a recent case, an acute outbreak with lighting up of fresh joints seemed definitely associated with an obstinate ulcer of the arm which involved the elbow joint. Attacks of cystitis and dysentery or an influenza may cause the recurrence of the previous disease. We see in the ostroarthropathy associated with bronchicetasis a condition which may have some bearing on this matter.

A difficult question to answer is as to the possibility of overgrowth of bone following on the changes which we have mentioned. Can hypertrophic bone changes, as we see shown in the so-called "osteoarthritic" type, occur with, or secondary to, these changes of the atrophic type? If this occurs, the identity of the two types is proved. The majority of the descriptions of the discase consider this to be the ordinary course, but recently some doubt has been cast on this opinion. I know of no definite pathologic reports which will settle the matter.

The Hypertrophic Type--Here one of the prominent features is the bony outgrowth. The majority of the classical descriptions of the disease refer to this condition. The cartilage as a rule shows very marked involvement, with erosion and atrophy of the joint surface, while proliferation is occurring around the edges. This outgrowth of cartilage may become converted into bone, often very irregularly. Bannatyne states that as a rule these outgrowths have a covering of cartilage. We are all familiar with the appearance of the bones from this type of disease, although characteristic instances probably are secn clinically very much less frequently than those of the other type. In the joints of the limbs there may be changes in the bones, but in the spine the bone may be little affected. Some cases of this type may be very acute.

There are certain manifestations of the disease which deserve special mention. These are: 1 , The conditions in the spine; 2 . the monarticular type; 3 , the so-called Still's disease.

Arthritis Deformans in the Spine.-We may have either type of the disease occurring in the vertebræ. Some involvement of the cervical region is very common in the atrophic type. This, however, rarely results in very marked permanent change. The frequency of the atrophic type in the lower spine is difficult to determine. Where we have definite bony outgrowth and ankylosis the process would seem to be of the hypertrophic type. The pathologic changes consist especially in the deposit of new bone. This occurs in very different positions. The intervertebral dises may be converted into bone, so that we have really a solid bony column, or the deposit may be largely in the ligaments, or, more uncommonly. there may be associated bony ankylosis of some of the smaller joints. These are very variable in position and extent; the bony deposit is very rarely symmetrical on the two sides. It may be on one side alone, or slight on one side and very marked on the other. The anterior ligaments may be ossified for a considerable distance and perhaps only one intervertebral articulation.

This irregularity of bony deposit is very striking when one examines a number of specimens, and is comparable to the irregular conditions found in the peripheral joints. The usual description given is that the condition begins in the articulations and extends to the ligaments, but signs of involvement of the joints do not seem to be nearly so common in museum specimens as the deposits in the ligaments.

These changes in the spine mav occur alone. but are frequently found associated with changes in the peripheral joints, and these of both types. This seems a strong argument for the unity of the disease. Two distinct diseases may coexist, but is such frequent coexistence probable? The cases showing definite bony outgrowths in the spine and the atrophic type of change in the peripheral joints are by no means rare. One association of special interest is that of the spinal type, with the so-called monarticular form as seen in the shoulder or hip joint. These latter, especially in the shoulder joint, are often very definitely atrophic.

There is much confusion regarding these spinal forms. We have the terms "Die chronische Steifigkeit der Wirgelsäule" (Bechterew), "Die chronische ankylosierende Entzundüng der Wirbelsäule" (Strümpell) and "Spondylose rhizomélique" (Marie), which probably are all designations for the same condition. This seems to be arthritis deformans of the spine. The varying types described depend on the condition and especially on the amount of involvement of the large peripheral joints. Bechterew, laying special stress on the associated conditions beginning with pain, sensory changes, muscular wasting, contractures, etc., regards the process as arising primarily in the nervous system. Marie, in the description of his type, insists on the condition of ankylosis of the vertebræ with ankylosis of the hips. Many variations have becn described, but it seems quite possible that these may be al] with one underlying condition. Cases are seen under observation at first with involvement only of the spine, and later one or both hips or shoulders concerned. I have had cases of spinal ankylosis with one, two, three or four of these large joints involved These various degrees of involvement are against there being distinct diseases.

Certain secondary changes are common; the nerve roots are frequently involved, whether by communicated inflanmation or direct pressure. This is seen especially in the cases regarded as sciatica, which are often due to arthritis deformans of the spine.

The most reasonable view at present seems to consider these various spinal forms as differing manifestations of arthritis deformans.

The Monarticular Group.--This has certain rather characteristic features. It occurs usually in advanced life. The joint involvement is in one of the shoulders or one of the hips. The malady known as morbus coxa senilis belongs here. The disability and the severe neuritis associated with it are important points. There are at times marked vasomotor phenomena seen in the affected limb. Pathologically, it would seem that these cases may belong to either type. In the shoulder joint the atroplic condition scems to predominate, while in the hip joint we have marked osteophytic growth. This, too, may be present in one part of the joint with very marked atrophy of other parts. The term monarticular is convenient to use as a description of a clinical type but perhaps unfortunate if it suggests anything distinctive from other forms of the disease.

'The identity of this type is emphasized by the fact that if careful examination be made slight involvement of other joints can often be found. This may be nothing more than the presence of Heberden's nodes or a slight involvement of possibly one knee joint or a finger or two, but the association is always suggestive.

In a recent very typical case there was involvement of one shoulder, with marked wasting of the shoulder girdle and very severe pain. The case was regarded as a typical instance of the monarticular form. The acute features gradually subsided, and the patient was nearly 
well, when suddenly the same process began very acutely in the other shoulder. Such instances speak against any special pecularity of this type. The cases may become polyarticular.

The So-Called Still's Disease.-There is some question as to what condition is meant by this term. The most remarkable view seems to be that the cases are examples of the polyarticular type of arthritis deformans occurring in children. They show certain characteristics -polyarthritis, general glandular enlargement and an enlarged spleen. In many of the cases the course is progressively downward.

Some writers would make a separate class of these cases, but where is one to draw the line?

When one sees a series of such cases at ages varying from two to twenty years, with the characteristic features present, but as the patient grows older glandular enlargement and the splenic enlargement less marked, although present, the inference seems justified that they are all examples of the same condition somewhat modified by age. I have no hesitation in saying that patients at fifteen with this condition have arthritis deformans; why not the same at the age of five? The occurrence of adherent pericardium in a large number of younger patients is of interest. It is difficult to explain, and probably has to be loft as one of the points to be solved.

The evidence at present seems to point to the unity of the various processes described. There is no group which can be constantly separated. Combinations of various features are common. Thus at present I have under observation a case in a young man with pronounced hypertrophic features, with marked general glandular enlargement and enlargement of the spleen. Trophic and hypertrophic changes may occur in the same joint, or one joint may show one type and another joint the other. In one case the atrophic changes are most marked, in another the hypertrophic. Why one process should predominate in a given case we do not know.

Certain writers would make another type which they term "infective." In the present condition of our knowledge it seems better to restrict this term to conditions associated with a definite organism.

\section{ETIOLOGY.}

Regarding this, one can only discuss the theories advanced without stating any definite conclusion. As already noted, there are probably three main theories to be considered: 1, That the disease is of neural origin; 2 . that it is a definite infection ; 3 , that it may be the result of various infections.

Neural Origin.-This dates back for a considerable period and has received very strong support in various quarters. The principal points brought forward in support of this view are as follows: Firstly, the symmetry of the lesions and their progression from the peripheral joints to those nearer the body; secondly, the similarity of the joint conditions to those which occur in association with certain disorders of the central nervous system, such as locomotor ataxia or syringomyelia; thirdly, the very marked associated muscular atrophy, the sensory disturbances, whether as regards pain or anesthesia, various trophic disturbances, such as glossy skin, the condition resembling scleroderma, etc. ; fourthly, the occurrence of neuritis

Symmetry of Lesions.-In looking into these conditions strong exception may be taken as to the value of certain of them. Thus we find, on carefully studying a series of cases, that the lesions are by no means necessarily symmetrical; in fact, in many instances, quite the contrary. While in many they do begin in the peripherai joints, very often the larger joints are attacked first.

similarty of Joint Changes.-In regard to the mat. ter of similarity to the joint changes in locomotor ataxia is this not true only of the latter stages of the lesions of arthritis deformans? Again, is the course of the disease as studied in a large series of cases in agreement with this view? It appears to me not. 'The cases of arthritis deformans proceeding to absolute destruction of the joints are, after all, comparatively few, and a great many proceed to almost complete recovery. Such a dif. ference does not seem to me to at all support the analog. between the two conditions.

Muscular and Sensory Disturbances.-The question of the muscular atrophy is a difficult one to explain. It does apparently occur with greater rapidity than is usua: in other forms of arthritis. As to a satisfactory explana. tion, I do not know that we are able to supply one. Thr various trophic disturbances may depend on the fre. quently associated condition of neuritis. This, in man! of the cases, is very well marked. Undoubtedly in some it is due to a communicated inflammation. In others it is probably the result of direct pressure, as is seen sometimes in the spinal forms.

Neuritis.-The very marked neuritis sometimes found in association with the monarticular types is at times very acute. 'The explanation is probably that it is often due to direct extension. In certain cases we may sug. gest the possibility of its being toxic.

When we turn to evidence to be obtained from the ex. amination of the central nervous system there is very little to support this view. In a small number of cases atrophic conditions have been found in the cells of the anterior horns. They are usually in the motor ganglion cells, but have not been extensive enough to support the idea that they are important factors in the disease. In the peripheral nerves in certain instances neuritis is found, but as this is not constant we can not consider it an etiologic factor. It is more probably secondary.

The view that reflex conditions may be the cause does not seem to me sufficiently supported by any evidence wr have at present. There is no evidence that any reflex action could cause the joint conditions we find.

There are some associated conditions of great interest which have been especially emphasized by Jones. Thus the occurrence with Raynaud's disease, exophthalmic goiter, etc., is interesting. In some cases one sees a condition of the skin much like scleroderma.

Specific Infection.-At present this seems to be the most reasonable view as to the etiologic factor. The character of the attack in many cases suggests an acute infection. The onset is frequently sudden, the symptoms may subside, to recur as suddenly, or may gradually progress. The subsequent course may be to complete recovery from that attack or may be to total loss of function of the affected joints; this, however, it is important to remember, being often due to secondary changes. With the acute symptoms are usually swelling, heat, redness and pain in the joints, some fever and often leucocytosis.

Visceral complications are rare, and yet in two recent cases with the joint symptoms the patients had acute pleurisy ushered in by a severe shaking chill. In one case a subsequent absence of reaction to tuberculin may be considered to rule out tuberculosis. With the acute features, marked enlargement of the glands in relation to the affected joints is almost invariably found. In the younger patients enlargement of the spleen may occur 
and this in cases not considered as examples of still: disease. Do these not suggest an infection very strongiy?

It may be noted that the glandular and splenic enlargement may occur with both types of the disease. The conditions in the nervous system may be explained by the action of toxins. When we turn to examine the results of cultural work, there is little as yet to show. Various observers have reported organisms (Schüller. Bannatyne, Wohlmann and Blaxall, Chauffard and Raymond, Van Dungen and Schmide, etc.), but the most important findings seem to be those of Poynton and Paine. ${ }^{2}$ From the knee joint of a man showing osteoarthritic changes they obtained a diplococcus which caused arthritis in a rabbit, with ulceration of the cartilage, flattening of the articular surfaces and the production of osteophytes with lipping of the joint. Such a result is certainly most suggestive. The results of cultures in the Johns Hopkins Hospital have thus far been negative.

Various Infections.-This view is a tempting one in many ways. As one organism may set up very different changes, so various organisms may cause the same changes. 'The association of arthritis deformans with various processes, such as pyorrhea, cystitis, dysentery, is suggestive. Tonsillitis and pyorrhea have by some been suggested as important sources of infection. In this series tonsillitis has been infrequent, while pyorrhea is so common that one hesitates to attach great importance to it as a causal factor. In rare cases the treatment of such local conditions has resulted in marked improvement in the general condition.

Whether these may be causal can not be proved, but they certainly at times scem to be definitely associated with exacerbations. This is not invariably the case. Some patients go through intercurrent infections without any change in the arthritis, or may even seem to be better for a time. This is true not only of septic processes, but also of infections such as influenza, measles. mumps, etc. Some would make an association between acute articular rheumatism and arthritis deformans in this way. Gonorrhea has by many observers been held to have a special relationship. In our cases, however. this has not been the case.

\section{STATISTICS OF ONE'HUNDRED AND SEVENTY CASES.}

In a series of 170 cases, among the acute infections in the previous history, measles was the most common, viz., in 93 instances. Following this came whooping cough in 43 , mumps in 31 , scarlet fever in 27 , malaria in 26 , typhoid fever in 20, chicken-pox in 18, influenza in 15, pneumonia in 16, diphtheria in 11, erysipelas in 3 and smallpox in 2. Recurring attacks of tonsillitis were infrequent, being noted in 7 cases. Various conditions. such as otitis media, pleurisy, carbuncles, dysentery, cystitis, etc., were noted in one or two instances. There was a previous history of gonorrhea in 23 cases, or 13 per cent. There was a history of syphilis in 5, and chancroid in 1 . It does not seem possible to speak with any certainty at present regarding the exact relationship of these many infections to arthritis deformans.

Regarding the various factors of incidence, the figures are as follows:

Family History.-There was a definite family history of arthritis in 62 cases. Of these, 15 were undoubtedly arthritis deformans, 35 were termed "rheumatism," and undoubtedly many of these were arthritis deformans; 1 was given as gout, and in 11 the nature was unknown. In some the family history of arthritis deformans was

\footnotetext{
2. Trans of the Path. Soc of London, vol, llit, 1902, p. 221.
}

remarkable. One patient's tather had the disease in a severe form, three brothers and three sisters suffered with it, all having chronic changes; only one sister had escaped. 'The patient's mother had escaped, but in her family the disease was common, all her brothers and sisters having it. Her mother also suffered with it. The combination of two such arthritic stocks would seem to portend disaster for the offspring. In another case there was a family history of the disease for four generations. In patients with a definite family history the disease does not seem to appear at an earlier age than in the others. There was a family history of tuberculosis in 39 cases.

Sex.-The males were in the majority- 89 males and 81 females. This is unusual in a large series, the females usually being in the majority. Many series give as high as 80 per cent. of females.

Race.-The white numbered 161, the colored 9 . The colored show a marked relative less susceptibility to the disease. The proportion of white to colored in the hospital is 7 to 1 , while the proportion of cases of arthritie deformans is 16 to 1 . The negro is specially prone to gonorrhea, tuberculosis and acute articular rheumatism, a point of interest in regard to their association witb arthritis deformans.

Occupation.-The largest number were engaged in housework, namely, 64; in other indoor occupations there were 46. At outdoor work there were 33. There was a surprisingly small number engaged in occupations involving special exposure. Neither exposure nor trauma seemed to play an important part as etiologic factors in this series.

Age.-On admission this was:

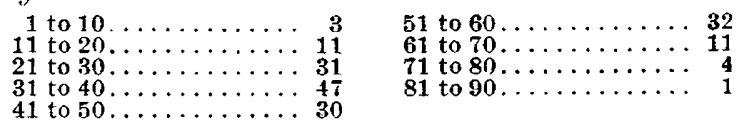

It is to be noted that 92 were below 40 years of age and 45 below 30 .

The age at onset, in 165 cases, shows a rather different distribution :

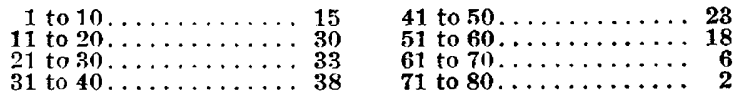

Practically in 70 per cent the onset was before the age of 40 , while in nearly one-half it was before the age of 30 years. This seems important, as so often the disease is considered to usually occur in late adult life.

Alcohol.-Fifty-one had used alcohol, the majority in moderation. About 10 might be described as heavy drinkers.

Pregnancy and Miscarriage.-While in some series these seem to have an important relationship to the onset of the disease, such was not the case in this clinic. Frequent repeated childbearing had occurred in some. but these are not numerous enough to be important. In only three of the women had the disease appeared soon after confinement. Uterine conditions were infrequent. Mental worry or anxiety did not seem to have played any part as an etiologic factor.

\section{CONCLUSIONS.}

'The conclusions which may be made are as follows:

1. That there are two great types of cases, the atrophic and hypertrophic, which are probably varying manifestations of the same disease.

2. That there are many variations, such as the spinal forms, the so-called monarticular form, the form occurring in children, etc., which are probably all arthritis deformans 
3. That the etiologic factor is not determined, but our present knowledge points to its being an infection of some nature.

4. 'That no opportunity of pathologic and bacteriologic examination should be neglected.

\section{AR'THRITIS DEFORMANS. * \\ JAMES J. WALSH, PI.D., M.D. NEW YORK CITY.}

At last light seems to be finding its way in among the obseurities of the rheumatism problem. Acute articular rheumatism is a microbic disease, one of whose principal features is its tendency to get well entirely except for the mark it may leave on the heart. Uric acid has nothing to do with rheumatic conditions, and is only a fetish that was set up to be worshipped by those who wanted to find scientific explanations for disease long before physiologic chemistry was ready to furnish any such explanation. Rheumatic gout now disappears, there is no middle term between gout and rheumatism, and the two diseases are not closely related at any point. Chronic rheumatism is a vanishing ailment, scen more and more rarely as greater care is used in diagnosis, and in the recognition of various painful nervous conditions with which it was formerly confounded.

'This preliminary clearing of the field leavs us, however, with a group of diseases, with regard to which as yet only abortive attempts have been made to get at a definite classification Under this group of diseases are found all those in which there is a deformity of joints usually progressive and not due to traumatism or known infection. The name selected for this group of diseases, arthritis deformans, is sufficiently noncommittal to be acceptable. The affections are certainly deforming. The underlying pathologic condition can be regarded, as a rule, as of inflammatory origin, though the piocesses of the inflammation run from acute, almost fulminant, to the extremely chronic type, which may take years before serious deformity results.

It may be said that it is only in recent years that attention has been especially called to this group of diseases. Physicians now see them frequently, and while a few years ago they were considered to be examples of a rather rare disease, this is due to the fact that in most cases the deformities when seen were attributed either to chronic rheumatism or to gout. The differential diagnosis has come for the most part within the last twenty-five years.

Arthritis deformans itself, however, in some form can be traced in history at nearly all times. The bones from the bodies of persons probably 5,500 years ago show in certain cases the signs of changes due to arthritis deformans. Bland Sutton described the same lesions as occurring in a mummy, taken from an Egyptian tomb, and which had been buried about 2,000 years before Christ. Virchow, excavating the bones from the cemetery of a medieval monastery, found similar conditions. It has even been said that the distinguished Italian painter of the Renaissance, Botticelli, always painted ugly hands, As a matter of fact, however, carcful examination of his pictures will show that his favorite model seems to have suffered from arthritis deformans, since the hands show signs of this disease, and

* Read at the Fifty-fifth Annual Session of the American Med. lcal Association. in the Section on Practice of Medlcine and approved for publication by the Fxecutive Committee: Drs. J. M. Anders, Frank Jones and W. S. Thayer. the reason for the ugly hands in his pictures is that, just as for her hollow chest, he followed this model too closely.

Arthritis deformans is, then, a veri old disease. It has existed continuously and it occurs much more frequently than has been thought. The affection of the spine, which is due to this disease and which is often spoken of as very rare, has been traced by Dr. Ruhrah ${ }^{1}$ down the centuries from the earliest times in Egypt, and any one who sees many cases in a city dispensary and hospital is sure to see one or more examples of the disease every year.

\section{TYPIS OF THE DISEASE.}

Such distinguished English authorities as Dr. Hale White, Dr. Garrod and Dr. Bannatyne divide the group of diseases commonly described under the name arthritis deformans into three types. These types may be briefly described as folıows: 'I'here is, first, the disease commonly known as osteoartluritis, often confined to the hip joint, and most common in old men. It consists of a bending of the neck of the femur and some other bony deformities connected with the hip joint. Secondly, there is the disease in its milder forms, often not recognized as a special affection, but considered as the resuit of certain linds of work or as of traumatic origin, which is most common in women in middle life, and is represented by deformities occurring in the terminal joints of the thumb, though in this the terminal joint is not necessarily always first affected. Dr. Hale White says that the deformity becomes considerable in these cases, and is ultimately largely due to bony outgrowth. "In a large number of cases the trouble gradually spreads until many joints are implicated and the woman may become a helpless cripple, and although in one sense she is very severely ill, yet the disease is very slow, the symptoms are not acute, and there is no pyrexia."

Whether the affection thus described is different from what is ordinarily called Heberden's nodes is hard to say. There seem to be cases in which a small amount of bony outgrowth on the terminal phalanges of two or three fingers does not progress beyond a certain point of disfigurement, and gives scarcely any discomfort except when there is some trauma; this, however, is not infrequent, because of the awkwardness induced by the deformity. According to a long-established English medical tradition, Ileberden's nodes occur especially in long-lived people. Whether there may be several groups of these nodular affections of the terminal joints of the fingers remains to be seen. Some of the cases certainly belong to an abortive type, and do not progress beyond a certain degree of awkwardness and unsightliness without further discomfort.

The third form of arthritis deformans is the one of most interest, since it attacks young people, simulates ordinary acute rheumatism, produces lasting changes in joints, has a definite tendency to recurrence, and ultimately makes its victim a helpless and pitiable cripple. The disease is more common in women than in men, occurring usually in young women, as Hale White says, about the age of 20 .

This affection is distinctly progressive. While it is markedly symmetrical in the two hands, it is likely to invade the wrists and then other joints, and the same thing may happen in the legs.

This is the form of arthritis deformans which, be-

1. The Amer. Jour. Med. Sclences, November. 1903 